Eur J Nucl Med Mol Imaging (2005) 32:511-512

DOI 10.1007/s00259-004-1726-3

Published online: 2 February 2005

(C) Springer-Verlag 2005

\section{Pre-therapeutic dosimetry with radiolabelled somatostatin analogues in patients with advanced neuroendocrine tumours}

\section{Dear Sir,}

Helisch and co-workers have reported important data on pre-therapeutic dosimetry with radiolabelled somatostatin analogues in patients with advanced neuroendocrine tumours (Eur J Nucl Med Mol Imaging 2004;31:1386-1392). They compared the dosimetry and biodistribution of ${ }^{86} \mathrm{Y}$ DOTA-D-Phe ${ }^{1}-\mathrm{Tyr}^{3}$ ]-octreotide (DOTA-TOC) with those of ${ }^{111} \mathrm{In}$-pentetreotide. They concluded that adequate dosimetry prior to ${ }^{90}$ Y-DOTA-TOC therapy is mandatory and that dosimetry with both compounds is possible, although dosimetry with ${ }^{86} \mathrm{Y}$-DOTA-TOC is more adequate. The latter finding is not surprising since ${ }^{86} \mathrm{Y}$ is the almost perfect surrogate for ${ }^{90} \mathrm{Y}$ and since dosimetry with ${ }^{86} \mathrm{Y}$ DOTA-TOC was defined as the gold standard by the authors.

The authors point out that dosimetry with ${ }^{86}$ Y-DOTATOC is not widely available owing to the limited access to the radionuclide and the elaborate correction algorithm required. Therefore in our opinion the aim should be to find a more widely feasible method for pre-therapeutic or, as we suggest, peri-therapeutic dosimetry. Since the aim is an effective treatment with ${ }^{90}$ Y-DOTA-TOC, we think that dosimetry on planar scintigraphic images should be performed with DOTA-TOC. It is known that the two peptides pentetreotide and DOTA-TOC have different affinity profiles on somatostatin receptors $[1,2]$. If pre-therapeutic dosimetry is demanded by an ethical committee or by authorities, we suggest the use of ${ }^{111}$ In-DOTA-TOC if ${ }^{86} \mathrm{Y}$ DOTA-D-Phe ${ }^{1}$-Tyr ${ }^{3}$-octreotide is not available.

The treatment protocol presented by the authors foresees three treatment cycles with a fixed injected activity of 4.4 GBq during each session. This seems surprising since most trials using ${ }^{90}$ Y-DOTA-TOC base the dose on body surface area or use a kidney absorbed dose adapted scheme with dosimetric calculations during the first therapy cycle [3-7]. It is also known that the kidney volume plays a

An authors' reply to this letter is available at http://dx.doi.org/10.1007/s00259-004-1727-2. crucial role in the absorbed dose to the organ [8]. Therefore an individually adapted dose scheme, based on body surface area or individual dosimetry, should be chosen. If individual dosimetry is performed in every patient and if several treatment cycles are planned, dosimetry would be most adequate when performed during the first treatment cycle by adding ${ }^{111}$ In-DOTA-TOC. Pre-therapeutic dosimetry would then be unnecessary.

A careful reading of this article conveys the impression that the main message is to exclude from treatment those patients who would receive an absorbed dose to the kidney higher than $27 \mathrm{~Gy}$. This would deprive them of a palliative treatment that also can potentially prolong life and improve its quality $[3,9]$. The fact that these cancer patients usually have no other treatment options makes their exclusion from treatment ethically unacceptable. Knowing the absorbed dose to the kidney (mGy/MBq), it is obvious that these patients can also be treated safely by a reduction in the injected activity.

The authors state correctly that no clear data exist on the relation between absorbed dose and irreversible renal toxicity in internal radiotherapy. For us this seems to be another argument as to why 27 Gy can only be a guideline and should not serve as a justification to exclude a patient from treatment with an injected activity adjusted to give a kidney dose of $<27$ Gy. For instance, 23 Gy was taken as the maximum allowed dose in another study [10]. Moreover, the pretherapeutic kidney function of the patient has to be taken into consideration. Of the patients we see, some have already had treatments with potentially nephrotoxic agents such as cisplatin or ${ }^{131}$ I-MIBG. An additional effect of toxicity is most likely.

It has been shown that the distribution of radiolabelled somatostatin analogues in the kidney is inhomogeneous [11]. The kidney damage observed after internal radiotherapy is located in glomeruli showing thrombotic microangiopathy [12]. These facts indicate that kidney dosimetry is much more complex than the way in which it is handled by the MIRDOSE concept.

To conclude, the dosimetric results of this work are important and we are convinced that internal radiotherapy has to be improved by means of dosimetry. But patients should not be excluded from a beneficial treatment by adherence to strict settings which are not proven and can be overcome. If individual dosimetry is performed, we suggest that this should be done peri-therapeutically during the first treatment cycle with ${ }^{111}$ In- 90 Y-DOTA-TOC. This is the most practical method and also the most convenient method for patients. The circumstances really represent the therapeutic situation. The "commercial success" of Zevalin in countries where pretherapeutic dosimetry is demanded might give us a hint as to where additional examinations may lead. 


\section{F. Forrer ( ), J. Mueller-Brand}

Institute of Nuclear Medicine, University Hospital Basel,

Petersgraben 4, 4031 Basel, Switzerland

e-mail: fforrer@uhbs.ch

\section{H. Maecke}

Division of Radiological Chemistry, University Hospital Basel, Basel, Switzerland

\section{References}

1. Reubi JC, Schar JC, Waser B, Wenger S, Heppeler A, Schmitt JS, et al. Affinity profiles for human somatostatin receptor subtypes SST1-SST5 of somatostatin radiotracers selected for scintigraphic and radiotherapeutic use. Eur J Nucl Med 2000;27(3):273-82.

2. Froidevaux S, Heppeler A, Eberle AN, Meier AM, Hausler M, Beglinger $\mathrm{C}$, et al. Preclinical comparison in AR4-2J tumorbearing mice of four radiolabeled 1,4,7,10-tetraazacyclododecane1,4,7,10-tetraacetic acid-somatostatin analogs for tumor diagnosis and internal radiotherapy. Endocrinology 2000;141(9):3304-12.

3. Waldherr C, Pless M, Maecke H, Schumacher T, Crazzolara A Nitzsche E, et al. Tumor response and clincical benefit in neuroendocrine tumors after $7.4 \mathrm{GBq}{ }^{90}$ Y-DOTATOC. J Nucl Med 2002:43:610-6.

4. Paganelli G, Bodei L, Handkiewicz Junak D, Rocca P, Papi S, Lopera Sierra M, et al. ${ }^{90}$ Y-DOTA-D-Phe ${ }^{1}$-Try ${ }^{3}$-octreotide in therapy of neuroendocrine malignancies. Biopolymers 2002;66:393-8.
5. Otte A, Herrmann R, Heppeler A, Behe M, Jermann E, Powell P, et al. Yttrium-90 DOTATOC: first clinical results. Eur J Nucl Med 1999;26:1439-47.

6. Waldherr C, Pless M, Maecke HR, Haldemann A, Mueller-Brand J. The clinical value of $\left[{ }^{90} \mathrm{Y}-\mathrm{DOTA}\right]-\mathrm{D}-\mathrm{Phe}^{1}-\mathrm{Tyr}^{3}$-octreotide $\left({ }^{90} \mathrm{Y}\right.$ DOTATOC) in the treatment of neuroendocrine tumours: a clinical phase II study. Ann Oncol 2001;12(7):941-5.

7. Kwekkeboom D, Bakker W, Kam BLR, Teunissen J, Kooij P, Herder W, et al. Treatment of patients with gastro-entero-pancreatic (GEP) tumours with the novel radiolabelled somatostatin analogue $\left[{ }^{177}\right.$ Lu-DOTA $^{0}$, Tyr $\left.^{3}\right]$ octreotate. Eur J Nucl Med Mol Imaging 2003;30:417-22.

8. Konijnenberg MW. Is the renal dosimetry for $\left[{ }^{90} \mathrm{Y}^{-D O T A}{ }^{0}, \mathrm{Tyr}^{3}\right]$ octreotide accurate enough to predict thresholds for individual patients? Cancer Biother Radiopharm 2003;18(4): 619-25.

9. Teunissen J, Kwekkeboom D, Krenning E. Quality of life in patients with gastro-entero- pancreatic tumors treated with $\left[{ }^{177} \mathrm{Lu} \mathrm{DOTA}^{0}, \mathrm{Tyr}^{3}\right]$ octreotate. J Clin Oncol 2004;22:2724-9.

10. Jamar F, Barone R, Mathieu I, Walrand S, Labar D, Carlier P, et al. ${ }^{86}$ Y-DOTA ${ }^{0}$-D-Phe ${ }^{1}-$ Tyr $^{3}$-octreotide (SMT 487)-a phase 1 clinical study: pharmacokinetics, biodistribution and renal protective effect of different regimens of amino acid co-infusion. Eur J Nucl Med Mol Imaging 2003;30:510-8.

11. De Jong M, Valkema R, Van Gameren A, Van Boven H, Bex A, Van De Weyer EP, et al. Inhomogeneous localization of radioactivity in the human kidney after injection of $\left[{ }^{111}\right.$ In-DTPA $]$ octreotide. J Nucl Med 2004;45(7):1168-71.

12. Moll S, Nickeleit V, Mueller-Brand J, Brunner FP, Maecke HR, Mihatsch MJ. A new cause of renal thrombotic microangiopathy: yttrium-90-DOTATOC internal radiotherapy. Am J Kidney Dis 2001;37(4):847-51. 